\title{
Médiévales
}

Langues, Textes, Histoire

67 | automne 2014

Histoires de Bohême

\section{Les récits de voyage médiévaux originaires de Bohême : produits d'une société confessionnalisée ?}

Medieval Travelogues from Bohemia: Products of a Society Characterized by its Different Religious Communities?

Vojtěch Bažant et Jaroslav Svátek

\section{CpenEdition}

Journals

Édition électronique

URL : https://journals.openedition.org/medievales/7421

DOI : 10.4000/medievales.7421

ISSN : $1777-5892$

Éditeur

Presses universitaires de Vincennes

Édition imprimée

Date de publication : 31 décembre 2014

Pagination : 103-120

ISBN : 978-2-84292-422-5

ISSN : 0751-2708

\section{Référence électronique}

Vojtěch Bažant et Jaroslav Svátek, «Les récits de voyage médiévaux originaires de Bohême : produits d'une société confessionnalisée ? », Médiévales [En ligne], 67 | automne 2014, mis en ligne le 31 décembre 2016, consulté le 24 avril 2022. URL : http://journals.openedition.org/medievales/7421 ; DOI : https://doi.org/10.4000/medievales.7421 


\section{Les récits de voyage médiévaux originaires de Bohême: produits d'une société confessionnalisée?}

La production de récits de voyage en langue tchèque n'a commencé qu'au $\mathrm{Xv}^{\mathrm{e}}$ siècle $^{1}$. Leur nombre reste limité par rapport aux textes du même genre créés dans l'espace géographique et littéraire allemand. Avec une petite dizaine d'ouvrages, la langue tchèque est en revanche bien représentée en comparaison avec les autres langues slaves. Ces textes ne connaissent pas de règles strictes, ce qui est typique pour tous les genres de la littérature médiévale. En Bohême comme ailleurs, le récit de voyage est surtout défini par l'itinéraire et par la mémoire du voyageur ${ }^{2}$. C'est ainsi que notre corpus comprend toutes sortes de textes «viatiques» que nous voulons ici à la fois présenter et interpréter dans le contexte des structures culturelles et sociales de la Bohême du Moyen Âge tardif. Ces dernières sont entre autre marquées par un dédoublement confessionnel connu sous la notion du «royaume du double peuple», résultat de l'évolution politicoreligieuse des pays tchèques pendant la première moitié $\mathrm{du} \mathrm{xv}^{\mathrm{e}}$ siècle. Le terme de «confessionnalité», que l'on utilise dans ce contexte, ne fait pas référence à une «confessionnalisation» totale de la société, assez discutable encore pour la fin du Moyen Âge. Il désigne plutôt une auto-identification

1. Les débuts de ce genre en Bohême sont souvent associés au fameux voyageur et missionnaire Odoric de Pordenone par le biais d'une théorie de ses origines tchèques. Cette hypothèse n'est soutenue que par une seule mention postérieure (Ego, frater Odoricus Boemus de Foro Julii) dans un manuscrit conservé à Paris $\left(\mathrm{BnF}\right.$, lat.2584, $\left.\mathrm{f}^{\circ} 126 \mathrm{v}^{\circ}\right)$ et n'a aucune importance pour le caractère ni le contenu de l'ouvrage que ce missionnaire franciscain écrivit en latin. Pourtant l'historiographie tchèque soulignait parfois l'origine tchèque d'Odoric. La production littéraire du bas Moyen Âge en Bohême ne dispose pas non plus d'une adaptation de cet ouvrage clé (à comparer avec celle en France de JEAN DE Vignay, Les Merveilles d'Outremer. Traduction du XIve siècle du récit de voyage d'Odoric de Pordenone, éd. D. A.Trotter, Exeter, 1990).

2. J. Richard, Les Récits de voyages et de pèlerinages, Turnhout, 1981 (Typologie des sources du Moyen Âge occidental, 38). 
de certains individus avec le parti catholique ou utraquiste. Dans notre étude, nous nous concentrons sur l'apparition de cet aspect dans les textes viatiques ${ }^{3}$. La période de la «production autochtone» fut pourtant précédée par un premier éveil de l'intérêt du public tchèque pour des ouvrages de voyage étrangers, dont plusieurs exemples de traductions donnent la preuve documentaire. Comme cette phase de traduction servit, parallèlement, de base pour la création ultérieure, il est utile pour commencer de s'y arrêter quelque peu.

\section{Traductions}

L'entrée de la littérature de voyage dans le milieu tchèque est matérialisée par la traduction des deux récits de voyage les plus populaires du Moyen Âge. Il s'agit en premier lieu de la version anonyme du Devisement du monde de Marco Polo. On ne connaît qu'un seul manuscrit de cette adaptation, conservée dans un codex qui comprend aussi la traduction du récit de Jean de Mandeville 4 . Le «Mandeville tchèque», composé autour de 1400, est une œuvre de l'universitaire Laurent (Vavřinec) de Březová, membre de la cour du roi Venceslas IV. Dans l'introduction, Laurent formule l'objectif de sa traduction: "pour que les Tchèques soient aussi au courant de ce qui est écrit dans ces livres ${ }^{5} »$, ce qui est également le but de la version allemande d'Otto de Diemeringen que Laurent utilisa comme modèle. Puisque la version tchèque de Mandeville était conçue comme un voyage de reconnaissance d'un chevalier, le lecteur devait comprendre que sa patrie n'était pas aussi unique que le pensaient ceux qui ne s'étaient jamais rendus hors des frontières du royaume. On peut trouver également

3. Voir le résumé du débat sur la «confessionnalisation» chez J. Deventer, « 'Confessionalization'. A useful Theoretical Concept for the Study of Religion, Politics and Society in Early Modern East-Central Europe?», European Review of History, 11 (2004), p. 403-425; O. MARIN, «L'Église utraquiste, entre tradition médiévale et modernité confessionnelle: jalons pour un bilan historiographique», dans M.-M.DE CEvins éd., L'Europe centrale au seuil de la modernité: mutations sociales, religieuses et culturelles. Actes du colloque international de Fontevraud (mai 2009), Rennes, 2010, p. 171-188. Les aspects sur la confession dans le domaine de la noblesse tchèque ont été déjà analysés par R.NovotNý, "Die Konfessionalität des böhmischen und mährischen Adels in der Zeit der Regierung Die Kon . Zur Herrschaftspraxis eines europäischen Monarchen, Vienne-Cologne-Weimar, 2012,

4rague, Knihovna Národního Muzea (désormais abrégé en: KNM), ms. III E42. Pour l'édition, voir Marka Pavlova z Benátek Million, éd. J. V.PRÁŠEK, Prague, 1902. Les traductions de Mandeville eurent beaucoup plus de succès. Pour la vue générale de l'état de conservation des manuscrits, voir Cestopis t.zv. Mandevilla. Český překlad pořizený Vavrincem z Březové, éd. F. S̆IMEK, Prague, 1911.

5. Ibid., p. 2. ce motif dans d'autres récits de voyage tchèques ${ }^{6}$ ainsi que dans le contexte européen de ce genre. C'est par des mots semblables que le pèlerin Jean Adorno soulignait que «certains pensent, mais bien sottement [...], qu'il n'y a de patrie que la leur ${ }^{7} »$.

Dans cette perspective «mandevillienne», l'expérience de voyage portait les traits aventuriers et exotiques d'un «livre de merveilles», dont le genre plutôt libre nous permet de dresser des analogies narratives avec d'autres œuvres littéraires. C'est le cas du roman tchèque d'Alexandre (Román o Alexanderovi) en prose, écrit à la charnière des $\mathrm{XIV}^{\mathrm{e}}$ et $\mathrm{XV}^{\mathrm{e}}$ siècles, probablement dans le milieu de la cour de Venceslas $\mathrm{IV}^{8}$. À la différence d'autres adaptations du Pseudo-Callisthène, cette version abrégée met en relief les aventures fabuleuses de la campagne indienne d'Alexandre où l'expérience du voyage est comparable à celle de Mandeville. En allan vers le royaume de Porrus, le fameux conquérant rencontre les Amazones, les bêtes sauvages exotiques et le «peuple» des créatures à tête de cheval ou avec les lèvres sur la poitrine ${ }^{9}$. La pertinence de cette comparaison n'est pas soulignée seulement par le style narratif du voyage, mais aussi par les conditions matérielles de sa réception. En effet, le manuscrit A de la traduction de Mandeville se trouve dans le même codex que celle du roman d'Alexandre ${ }^{10}$. Des topoï similaires ainsi que des motifs merveilleux d'animaux parlants, d'épées magiques ou de montagnes magnétiques, traversent d'autres ouvrages littéraires provenant du milieu tchèque ou tchéco-allemand, tels que les romans sur Stilfried, Bruncvík et d'autres ${ }^{11}$. Il est donc clair que les premières traductions des récits de voyage appartiennent à la littérature de distraction, dont la vocation est à la fois courtoise et didactique. Le récit de voyage y joue un rôle spécifique de medium de la connaissance du monde situé au-delà des frontières du pays. De plus, l'imagination de l'Orient merveilleux est aussi perceptible dans le genre de l'instruction historique, par exemple dans la Chronique de Jean de

6. Martin Kabátník, Cesta z Čech do Jerusalema a Kaira r. 1491-92, éd. J. V. PréšEK, Prague, 1894, p. 36.

7. JEAN ADORNo, Itinéraire d'Anselme Adorno en Terre sainte (1470-1471), éd. J. HEERS et G.DE GroER, Paris, 1972, p. 27.

8. J. KoLÁR, «Román o Alexandru Velikém v souvislostech české středověké prózy», Listy Filologické, 105 (1982), p. 209-215; F. P.MAgoun et S.HARRISON THOMSON, «Kronika o Alexandru Velikém. A Czech Prose Translation of the Historia de Preliis», Speculum, 3 (1928), p. 204-217; pour l'édition du texte voir J.Kolár, Próza českého středověku, Prague, 1983, p. 21-147.

9. J. Kolár, Próza českého středověku..., p. 83 sq.

10. Prague, KNM, ms. II C 10

11. L'édition des romans se trouve chez J. Kolár, Próza českého středověku..., p. 149162 (O Štilfridovi) et p. 163-178 (O Bruncvíkovi). 
Marignol $^{12}$. L'auteur voyagea, en tant que légat pontifical, en Asie jusqu'à la ville de Pékin. À la requête de l'empereur Charles IV de Luxembourg, il composa la chronique de Bohême tout en l'intégrant à l'histoire générale du Salut. Les premiers chapitres de l'ouvrage, décrivant la Création du monde, comprennent des histoires fabuleuses sur les nations orientales et reflètent en même temps l'expérience personnelle de l'auteur-voyageur.

Malgré leur caractère propre-la fiction pour Mandeville,l'expérience réelle pour Marco Polo, la démarche historique pour Marignol -, ces ouvrages littéraires et leurs semblables avaient, à cette époque, la même finalité pour le milieu de la cour de Bohême. D'un côté, ils faisaient connaître la nouvelle réalité des pays lointains par l'intermédiaire d'ouvrages d'un style relativement nouveau qui mélangeait la fiction à la littérature savante (chronique du monde, géographie). De l'autre, par leur transposition en une langue vernaculaire (le tchèque, l'allemand), ces textes pouvaient satisfaire la demande des élites locales. Les commanditaires de ces ouvrages étaient souvent issus de familles de la haute noblesse qui était soit concentrée autour de la cour du souverain (et, de ce fait, liée étroitement à la ville par le biais des savants de l'Université), soit évoluait en dehors de ce milieu, créant des structures parallèles de pouvoir. Les ouvrages géographiques et historiques traduits en langue vernaculaire servaient alors à représenter le pouvoir et le statut social de leurs commanditaires et à les divertir, plutôt qu'à instruire un vaste public ignorant le latin. De toute façon, l'éclatement des guerres hussites mit un terme à cette période de création et ce n'est qu'après la phase des conflits (années 1420-1430) que le milieu tchèque connut les premiers récits authentiques, écrits par les voyageurs eux-mêmes. Comme nous l'avons déjà indiqué, ces textes naissaient dans le nouveau cadre social marqué par la rivalité des deux tendances confessionnelles - la catholique et l'utraquiste. Nous nous proposons donc d'étudier comment cet arrièreplan socioculturel a influençé les représentations narratives des voyageurs tchèques.

\section{Pèlerinages en Terre sainte}

Les textes sur les pèlerinages en Terre sainte représentent une source idéale pour l'étude du rôle que la confession a pu jouer dans leur rédaction. Des quatre récits conservés pour la deuxième moitié $\mathrm{du} \mathrm{xv}^{\mathrm{e}}$ siècle, deux ont été composés par des non-catholiques: Martin Křivoústý, prêtre utraquiste

12. Johannes de Marignola, Cronica, éd. J. Emler, (Fontes Rerum Bohemicarum III, Prague, 1882, p. 485-604). Il est symptomatique que le traducteur de Mandeville, Laurent de Březová, soit devenu plus tard l'éminent historiographe du mouvement hussite. Voir VAVŘINeC zBřezové, Kronika husitská, éd. J. Goll, FRB V, Prague, 1893, p. 327-542. dont le voyage date des années $1470^{13}$, et Martin Kabátník, qui fut présen en Palestine et en Égypte, en 1491-1492, comme membre de «l'Unité des frères », une branche radicale du mouvement de réforme en Bohême qui devint bientôt indépendante ${ }^{14}$. Tous les deux se rendirent en Terre sainte pou y chercher les restes de l'Église primitive à laquelle la réforme utraquiste faisait beaucoup référence. Leur effort était en partie motivé par la nécessité de trouver une légitimité aux nouveaux ensembles confessionnels dont les principes tendaient à la réforme de la foi et au retour à la vérité de l'Écriture, tout en rejetant la tradition de l'Église institutionnelle ${ }^{15}$.

À côté de ces deux textes, deux autres récits, beaucoup plus traditionnels par leur forme et contenu, sont nés dans le milieu tchèque: la liste d'indulgences du pèlerinage d'Henri (Jindřich) de Stráž, un noble de Bohême du Sud ${ }^{16}$, datée de 1445, et le récit intitulé Le Pèlerinage au Saint-Sépulcre du seigneur Jean Hasištejnský de Lobkovice ${ }^{17}$. La liste du pèlerinage d'Henri de Stráž, qui est d'ailleurs la plus vieille description de la Terre sainte en langue tchèque, se présente sous la forme d'un catalogue abrégé des sanctuaires et des lieux saints ${ }^{18}$. Ce n'est qu'après la liste normative du paysage sacré que suit la véritable description de la ville de Jérusalem ainsi que l'itinéraire même du voyage d'Henri. En revanche, le Pèlerinage au Saint-Sépulcre d'Hasištejnský se distingue par sa qualité stylistique; par sa forme, le récit est plus riche, plus vaste et d'une véritable qualité littéraire, ce qui le classe aux côtés des récits de son époque, tels que l'Evagatorium de Felix Fabri ou la Peregrinatio ad Terram sanctam de Bernard de Breydenbach.

Malgré la diversité formelle et la disparité socio-religieuse de leurs auteurs, les quatre récits présentent des traits communs, notamment sur le

3. J. Kolár, «České znění Cesty do Jeruzaléma Martina Křivoústého», Strahovská knihovna, 18-19 (1983-1984), p. 67-95.

14. Martin Kabátník, Cesta z Čech do Jerusalema.

15. B. MAŁYsZ, «Problematika wyznaniowa w relacjach Czechów podróżujących na bliski wschód w II połowie xv wieku», dans D.Quirin-PoPŁawsKa éd., Portolana. Studia mediterrenea II: Religie świata śródziemnomorskiego, Cracovie, 2006, p. 211-222.

16. Ce témoignage précieux se trouve encore dans un manuscrit non édité des Archives municipales de Prague (désormais abrégé en: AMP) : ms. $1865, \mathrm{f}^{\circ} 368 \mathrm{r}^{\circ}-370 \mathrm{r}^{\circ}$

17. Jan HasištejnskÝ z Lobrovic, Putování ksvatému hrobu, éd. F. Strejčé, Prague, 1902. Une partie de cet ouvrage, concernant la description de l'île de Rhodes, a été récemment traduite en français et intégrée dans le recueil J.-B.DE VAIVRE et L.VISSIËre éd., "Tous les deables d'enfer". Relations du siège de Rhodes par les Ottomans en 1480, Genève, 2014, p. 799-812. Â ce dernier ouvrage, on peut associer encore le récit d'Heinrich de Zedlitz, un p. 799-812. A ce dernier ouvrage, on peut associer encore le récit d'Heinrich de Zedlitz, un
Silésien qui voyageait en Palestine au bord du même bateau qu'Hasištejnský en 1493. Pour son édition, voir «Die Jerusalemfahrt des Heinrich von Zedlitz (1493)», éd. R. RöнRIchт, Zeitschrift des deutschen Palaestina-Vereins, 17 (1894), p. 98-114, 185-200 et 277-301.

18. Le problème de la tradition de localisation des lieux saints en Palestine est traité dans l'étude classique de M. HaLBwACHs, La Topographie légendaire des évangiles en Terre sainte, Paris, 1941. 
plan de la description générale de Jérusalem et de ses alentours. L'espace de la ville et du paysage sont «lus » d'après le modèle traditionnel localisant les événements bibliques sur les lieux de la dévotion des pèlerins. Seul Martin Křivoústý explique comment il approcha les sanctuaires de la Ville sainte: «J'ai inscrit toutes ces choses d'après leur position depuis la maison de David et d'après ce qu'ils nous ont montré, et non selon le temps et l'ordre des événements mêmes. ${ }^{19}$ » L'auteur du récit exprime ainsi la nécessité de défendre son style, qui ne respecte pas les Écritures mais l'itinéraire réel du pèlerinage.

L'extrait cité révèle un autre trait commun avec les récits tchèques: la perception de la Terre sainte est soumise à la stratégie des visites guidées, organisées par les franciscains du couvent du Mont-Sion. Ces gardiens de nombreux sanctuaires détenaient une sorte de monopole en ce domaine depuis les années 1330, grâce aux accords entre Robert de Naples et AnNâsir Muhammad, sultan de l'Égypte mamlûk ${ }^{20}$. Comme beaucoup d'autres témoignages des pèlerins de l'Europe latine, les récits des Tchèques racontent également comment les «déchaux» (bosáci, c'est-à-dire les franciscains) guidaient les visiteurs, célébraient les messes, invitaient les pèlerins à la prière et, surtout, leur expliquaient en plusieurs langues l'importance des lieux qu'ils visitaient ${ }^{21}$. En ce sens, ils définissaient l'orientation des pèlerins à Jérusalem et dans ses alentours.

La description des lieux visités est dans tous les cas suivie de passages avec une évaluation personnelle: l'aspect du paysage autour de la Ville sainte, la description des habitants, de leurs vies et de leurs mœurs, ainsi que leurs relations avec les chrétiens et les juifs, la situation politique, etc. Dans ces descriptions, les voyageurs quittaient mentalement le territoire sacré autour de Jérusalem. Parmi eux, Martin Kabátník fut le seul à le faire aussi physiquement. Il continua sa recherche des descendants de l'Église primitive en Égypte, et la description de ce pays est plus exotique et merveilleuse que celle de la Terre sainte ${ }^{22}$. Probablement fidèle à la forme canonique de la Bible, Kabátník ne mentionne pas les sanctuaires chrétiens du Caire et ne visite pas les endroits liés au culte de sainte Catherine (Alexandrie, couvent au mont Sinaï) ni les ermitages dans le désert au sud-est du pays. Sans prendre le village de Mataria, près du Caire, pour un lieu de l'enfance du Christ, il s'y rend seulement «pour voir le jardin où le baume croît ${ }^{23}$ ».

19. J. Kolár, «České znění Cesty do Jeruzaléma Martina Křivoústého... », p. 80.

20. N. Paulus, Geschichte des Ablasses im Mittelalter, t. II, Darmstadt, 2000, p. 243.

21. Jan HasištejnskÝ zLobrovic, Putování..., p. 58 .

22. Martin KabÁtNí, Cesta z Čech do Jerusalema..., p. 19-3.

23. Ibid., p. 26-27. Le voyageur tchèque ne mentionne pas un autre topos habituel: l'identification des pyramides aux greniers de Joseph. La seule référence, d'ailleurs assez curieuse, à ce héros vétérotestamentaire concerne le système de gouvernement mamlûk. Kabátník apprit que c'est grâce à Joseph et la prospérité de son gouvernement que, même à
Ainsi, la vénération des saints et le respect des apocryphes et des autres textes légendaires sont remplacés chez Kabátník par l'émerveillement pour l'autre.

Aucun désaccord dans le domaine de la foi ne se laisse lire lorsque l'on compare les quatre textes. Le refus du pèlerinage en tant que tel, trait caractéristique de certains courants radicaux de la réforme de Bohême ainsi que de la réforme luthérienne, n'est pas encore présent dans les récits utraquistes. Certains motifs du voyage sur les traces du Sauveur et la manière dont ils sont traités peuvent pourtant être considérés comme des «points de friction». Pour les non-catholiques, soit le frère Kabátník et l'utraquiste Křivoústý, les lieux saints représentaient l'espace de l'authenticité de l'Écriture, autorité suprême de leurs principes théologiques. Tandis que les théories radicales de la réforme en Bohême avaient mis en doute le culte des saints, la vénération des images ou le nombre excessif des fêtes de l'Église, la dévotion en Terre sainte s'adressait aux personnages de la Bible et à leurs actes. Bien que le milieu de la réforme du $\mathrm{xv}^{\mathrm{e}}$ siècle en Bohême ne reniât pas encore le culte marial comme le fit la réforme de Luther, les utraquistes, les taborites et les frères tchèques en étaient souvent accusés ${ }^{24}$. Les pèlerins non catholiques visitèrent donc les sanctuaires liés à la vie de la Vierge en les regardant avec attention, mais le récit du catholique Hasištejnský ainsi que le traité d'Henri de Stráž en parlent avec plus d'intensité. Les représentations du pèlerinage diffèrent plus nettement encore dans le cas de sainte Hélène. Alors que Křivoústý ne mentionne la mère de l'empereur Constantin qu'une seule fois et Kabátník pas du tout, Jean Hasištejnský y a constamment recours. En une occasion, il raconte comment elle avait «construit» la topographie légendaire de la Terre sainte : «Personne n'avai pu apprendre à sainte Hélène de façon certaine quelle était celle des deux pierres sur laquelle le Christ avait mis le pied. Elle les fit alors maçonner toutes deux dans la cave [de la maison de Pilate] ${ }^{25}$.» Pour les catholiques, le culte de sainte Hélène était fondé sur le recouvrement de la Vraie Croix et enrichi par la localisation des lieux de mémoire, la construction des églises et des chapelles. En revanche, pour un utraquiste ou un frère tchèque, il s'agissait d'une sainte postbiblique et aucun élément de son culte ne pouvait être prouvé par les Écritures.

Le sujet des indulgences accordées pour la visite des lieux saints en Palestine pouvait représenter un autre point de discorde entre les auteurs

son époque, les hautes fonctions administratives de l'Égypte n'étaient confiées qu'aux «gens achetés» (ibid., p. 20). Concernant le discours littéraire sur l'Égypte chez les voyageurs européens, voir J.Guérin DaLle Mese, L'Égypte. La Mémoire et le rêve. Itinéraires d'un voyage, 1320-1601, Florence, 1991, qui ne connaît malheureusement pas le récit du frère de Bohême.

24. O.Halama, Otázka svatých včeské reformaci, Prague, 2001, p. 56-77.

25. Jan HaSIštejNGKÝ ZLoBKovic, Putování.., p. 64. 
catholiques et leurs homologues utraquistes. Après son entrée dans l'espace des faits bibliques, Martin Křivoústý signale qu' «il y a beaucoup de lieux avec indulgences ${ }^{26}{ } »$, sans développer davantage ce constat ou continuer par l'énumération de ces lieux. Le frère Kabátník prend lui aussi conscience du système des indulgences dans le cadre de la «visite guidée» sous l'égide des franciscains: «Et, en tous les lieux, ils donnaient de grandes indulgences dont j'ignore la quantité. Car il y a, pour chaque lieu, des indulgences spéciales et elles se différencient les unes des autres, les petites des grandes ${ }^{27} \gg$. Par ces mots, l'auteur du récit semble plutôt marquer son indifférence, voire son ignorance, qu'émettre une condamnation morale de cette pratique que la théologie des hussites critiquait avec virulence.

Les indulgences jouent un rôle important et particulier dans le quatrième récit que nous avons laissé jusqu'ici de côté. La relation anonyme du pèlerinage d'Henri de Stráž porte le titre Ceci sont les indulgences de la Terre sainte, suivi par une liste normative des lieux saints aux alentours et à l'intérieur même de Jérusalem ${ }^{28}$. L'appartenance confessionnelle d'Henri de Stráž, acteur principal du pèlerinage, reste incertaine. Ce noble faisait partie de la société des seigneurs et clercs utraquistes, envoyés à Rome en 1446 pour obtenir la confirmation archiépiscopale pour Jean Rokycana ${ }^{29}$. L'existence de la liste d'indulgences attachée à son voyage semble témoigner au contraire de sa foi catholique. Or, un ajout, sur un mode sans doute ironique, à la fin du récit, met en question la vénération hypothétique des lieux saints ainsi que le respect de l'autorité de la liste d'indulgences: «Item, Jérusalem est un lieu saint, bien dévot et joyeux, avec de larges indulgences et du bon vin. Et qui obtient ces indulgences et boit ce vin, peut être gai jusqu'à la mort joyeuse de ce monde ${ }^{30} \gg$. D'autres sources n'attestent son inclination temporaire au catholicisme que sur le plan politique durant la période du règne d'Albert IV d'Autriche et de son fils, Ladislas le Posthume ${ }^{31}$. Enfin, pour illustrer l'appartenance confessionnelle d'Henri, on dispose encore d'une source curieuse. Il s'agit d'un épisode faisant partie des Vieilles annales de Bohême: en revenant de Terre sainte, Henri de Stráž s'arrêta à Constantinople où il était censé discuter avec

26. J. KoLÁR, «České znění Cesty do Jeruzaléma Martina Křivoústého...»»,p. 78

27. Martin Kabátník, Cesta z Čech do Jerusalema..., p. 13.

28. Parmi de nombreux parallèles de cette liste d'indulgences pour la Terre sainte, on peut trouver des voyageurs français. Voir J.SvÁTEK, Discours et récits de nobles voyageurs $\grave{a ̀ ~ l a ~ f i n ~ d u ~ M o y e n ~ A ̂ g e, ~ t h e ̀ s e ~ e n ~ c o t u t e l l e ~ s o u s ~ l a ~ d i r e c t i o n ~ d e ~ B . S c h n e r b ~ e t ~ M . N e j e d l y ́, ~}$ Université Lille III, 2012, p. 169-193.

29. Staré letopisy české, éd. J. Charvát, Prague, 1941 (Dílo Františka Palackého 2), p. 132-133.

30. Prague, AMP, ms. $1865, \mathrm{f}^{\circ} 368 \mathrm{r}^{\circ}$

31. Staré letopisy české, p. 110 (couronnement d'Albert par les nobles de Bohême parmi lesquels se trouvait aussi Henri de Stráž). le sultan ottoman de la communion sous les deux espèces, rite liturgique pratiqué par les utraquistes. Le sultan lui répondit

«Moi, je préfère plutôt servir le Dieu qui donne à manger et à boire que celui qui donne seulement à manger, et ne donne rien à boire.» Après avoir entendu ce discours, le seigneur Henri rentra immédiatement à Prague, et communia sous les deux espèces jusqu'à sa mort. Car avant, il était [du parti de ceux qui communient] sous une [espèce] comme un véritable jednušk $a^{32}$.

Selon toute probabilité, il s'agissait pourtant d'une anecdote fictive au service de la propagande utraquiste ${ }^{33}$. Dans cette perspective, ce récit de pèlerinage apporte la preuve qu'Henri de Stráž, ou bien le copiste qui en rédigea le voyage, représentait un milieu intermédiaire, difficile à classer priori dans les catégories confessionnelles de la société des pays tchèques du Xve siècle.

La Terre sainte devint aussi l'un des sujets de prédilection des premiers incunables en langue tchèque. Ces textes prouvent également le renouvellement de l'intérêt pour les récits de voyages de provenance étrangère en Bohême. Dans les années 1490, l'imprimeur Nicolas (Mikuláš) Bakalář de Pilsen ${ }^{34}$ édita Le Traité de la Terre sainte ${ }^{35}$ et La Vie de Mahomet ${ }^{36}$. Les deux ouvrages sont de facto le traitement du premier récit de voyage imprimé de Bernard de Breydenbach, chanoine de Mayence ${ }^{37}$. La version tchèque $\mathrm{du}$ «traité» reprend à son modèle une partie abrégée décrivant la Terre sainte. De plus, par rapport aux originaux allemand et latin, elle est dotée d'un prologue qui nous informe sur le pèlerinage d'un certain chanoine de Ratisbonne, nommé Nicolas, et de Jean, bourgeois de Nuremberg. Ces pèlerins se rendirent en Terre sainte le lundi 6 mai 1482 «au nom de Dieu, pour le seul amour de Dieu et le salut de notre âme, et non par vaine gloire ou par une curiosité quelconque, afin de visiter les lieux

32. Ce mot, d'ailleurs intraduisible, désigne d'une façon ironique un catholique pratiquant sous une seule espèce (pod jednou zpưsobou).

33. Or cet épisode est daté de 1478 , soit dix-huit années après la mort du noble en question. De plus, il ne se trouve que dans les manuscrits postérieurs, du début du XVII ${ }^{e}$ siècle Staré letopisy české..., éd. J. Charvát, p. 185-186. Voir aussi B.Matysz, «Problematika wyznaniowa... », p. 212-213.

34. Z. V. TовоLKa, Plzeňský tiskař Mikuláš Bakalár. Písek, 1927; L. Kонút, Mikuláš Bakalár Štětina: štúdie a materiály o živote a diele slovenského prvotlačiara vPlzni, Bratislava, 1966.

35. Conservé en deux exemplaires: Prague, KNM, ms.25 E 8, et Prague, Královská kanonie premonstrátů na Strahově, ms. DR IV 37/e.

36. Prague, Královská kanonie premonstrátů na Strahově, ms. DR IV 37/c

37. Bernhard von Breydenbach, Peregrinatio in Terram sanctam, Mayence, $1486 . \mathrm{Ce}$ récit devint un véritable best-seller à la fin du $\mathrm{Xv}^{\mathrm{e}}$ siècle. Outre la première édition latine, on dispose des imprimés en allemand, français, espagnol, néerlandais et italien, tous de la fin du $\mathrm{XV}^{\mathrm{e}}$ et du début du $\mathrm{XVI}^{\mathrm{e}}$ siècle. 
saints, surtout les lieux et les pays où Notre Seigneur Jésus en sa sainte humanité vécut et fit des miracles, pour ensuite souffrir sa grande et cruelle passion, et daigner mourir pour notre salut ${ }^{38} »$. Ces motifs relient le désir du salut avec la culture mémorielle des pèlerins, commune à tous les auteurs mentionnés jusqu'ici. Bien que le texte tchèque s'identifiât pour la suite avec son modèle, certaines différences avec les originaux allemand et latin de Breydenbach peuvent y être repérées en ce qui concerne les détails de certaines indulgences auprès des sanctuaires en Terre sainte. Dans le cadre de cette description, l'auteur de la traduction insère une phrase stéréotypée, «ici sont les indulgences de VII ans et VII quarantaines », même là où elle est absente chez Breydenbach ${ }^{39}$. Est-il possible d'en tirer la conclusion que la Peregrinatio in Terram sanctam du chanoine de Mayence a servi de base textuelle pour une description du pèlerinage effectué en réalité par Nicolas de Ratisbonne et Jean de Nuremberg, mentionnés dans le prologue? Si tel est le cas, le Traité reste un témoignage unique et curieux du voyage des deux pèlerins allemands, conservé seulement en tchèque et non dans sa langue d'origine, qui était selon toute probabilité le haut allemand ${ }^{40}$.

Par rapport au Traité de la Terre sainte, la Vie de Mahomet représente moins un récit de voyage qu'une adaptation des parties de Breydenbach contenant non seulement la vie du Prophète, mais aussi l'énumération des nations qui habitaient la ville de Jérusalem ${ }^{41}$. L'auteur de la traduction tchèque, identifié par la recherche historique avec l'imprimeur lui-même, Nicolas Bakaláŕ de Pilsen, se présente en tant que catholique assez fervent. Preuve en sont les passages sur la communion sous les deux espèces, par lesquels Breydenbach caractérise certains chrétiens orientaux de Jérusalem; ceux-ci sont en effet totalement supprimés dans la version tchèque, car ils pouvaient gêner les croyances religieuses du traducteur et de son public. De même, le texte tchèque prend les «Indiens» (c'est-àdire des chrétiens d'Éthiopie) pour des «hérétiques», à la différence d'une caractérisation beaucoup plus modérée du modèle allemand ou latin. Dans le contexte de la production des récits de voyage en Bohême, le traducteur - et en même temps imprimeur - représente un pôle contraire à celui de

38. Prague, KNM, ms. 25 E $8, \mathrm{f}^{\circ} 1 \mathrm{v}^{\circ}$. La datation est correcte (en 1482, le 6 mai était un lundi) mais elle précède d'un an le voyage de Breydenbach, auteur du modèle textuel de notre traité.

39. Cette anomalie fut déjà signalée par L.KoutNíkovÁ, «Český prvotisk 'Traktát o zemi svaté'», Časopis československých knihovníkủ, 3 (1924), p. 191-196 (ici p. 194-195). D’après la comparaison des textes, il est aussi sûr que le traducteur a compilé son traité à partir de la version allemande (pour la plus grande part) et aussi latine de Breydenbach.

40. Cette hypothèse nous paraît plus probable que celle selon laquelle les deux pèlerins auraient été inventés par l'imprimeur lui-même (cf. L. KoutNíková, Český prvotisk..., p. 195$196)$.

41. V. SoкоL, «Život Mohamedův zr. 1498 a jeho předloha», Listy Filologické, 50 (1923), p. 35-42. ses contemporains, l'utraquiste Martin Křivoústý et le frère tchèque Martin Kabátník. La partie identique de la Vie de Mahomet concernant les chrétiens orientaux eut pourtant par la suite un destin complexe. En 1539, elle fut intégrée dans l'édition imprimée du récit de Kabátník, cette fois-ci avec les parties sur la communion sub utraque specie ${ }^{42}$. Cette double adaptation de Breydenbach représente donc un cas particulier de sa réception dans son contexte européen.

Malgré la différence dans la perception des traditions post-bibliques et dans l'octroi des indulgences, on pourrait conclure que le pèlerinage en Terre sainte et son reflet dans les récits de ses participants furent une affaire supra-confessionnelle. Il existe pourtant un dernier aspect, peut-être le plus important, qui montre la scission entre les auteurs utraquistes et leurs homologues catholiques dans ce domaine. Il s'agit de la perception différente des chrétiens orientaux. Comme nous l'avons déjà constaté, Martin Křivoústý et Martin Kabátník les observaient avec attention afin de leur trouver des traits communs avec la Bohême réformée, notamment la pratique de la communion sous les deux espèces. Dans leurs récits, les deux utraquistes donnent une image de la tolérance mutuelle entre les divers chrétiens de ce territoire sacré, respectée même par les franciscains. Kabátník raconte qu'après avoir révélé aux franciscains qu'il était venu du «pays lointain de Bohême», les «déchaux» l'accueillirent bien et, «en accordant toute grâce», lui donnèrent tout ce dont il avait besoin ${ }^{43}$. Son prédécesseur, Křivoústý, s'exprime sur le même ton en énumérant les nations habitant la ville de Jérusalem ${ }^{44}$.

Arméniens, Indiens, Syriaques, jacobites, Géorgiens, nestoriens, Grecs, Romains ou Latins qui, plutôt que refuser, estiment qu'il faut distribuer [la communion] à tous ceux qui le demandent avec dévotion. Et puisque toutes ces nations communiant au divin corps et au divin sang sous les deux espèces ne sont pas hérétiques, les Tchèques ne le sont pas non plus, mais sont des vrais fils de notre mère la sainte Église. Et communiant sous les deux espèces, ils le font par l'autorité du pape suprême, Notre Seigneur Jésus-Christ.

Ce qui est encore plus étonnant est que le prêtre utraquiste écrit avoir été autorisé à célébrer trois fois la messe à l'intérieur de l'église du SaintSépulcre. Sans préciser les détails de la liturgie, il indique que l'office

42. Ibid., p. 42.

43. Martin KabátNí, Cesta z Čech do Jerusalema..., p. 17

44. J. Kolár, «České znění Cesty do Jeruzaléma Martina Křivoústého...», p. 82. 
avait lieu sur la pierre de la Déposition du Christ, endroit appartenant aux Arméniens ${ }^{45}$.

Malgré cette ambiance paisible entre les différents rites ${ }^{46}$, le récit de Křivoústý donne à voir des cas d'intolérance envers d'autres pratiques liturgiques:

Dans cette église, les chrétiens de toutes les nations possèdent leurs lieux pour y célébrer les messes d'après leur coutume sans aucun obstacle. Mais les nôtres, c'est-à-dire les Latins, diffament tous les autres en les tenant pour hérétiques (et vice versa). Les uns n'empêchent toutefois pas les autres de célébrer leurs offices ${ }^{47}$

Il est très probable que l'utraquiste ne pensait pas seulement aux tensions à l'intérieur de la Terre sainte. Car l'expression «les nôtres, c'est-àdire les Latins », désigne plutôt les catholiques de sa patrie. Cette hypothèse est bien confirmée par la description des nations hiérosolymitaines qu'a faite son contemporain, le noble catholique Jean Hasištejnský de Lobkovice:

ll est à savoir qu'une huitaine de chrétiens [...] résident dans l'église du Saint-Sépulcre. Or, aucun d'eux n'est chrétien sauf les déchaux [bosáci]. Car parmi tous les autres, qui s'appellent chrétiens et en revendiquent le nom, chaque secte tient certains articles hérétiques contre la foi chrétienne universelle.

Il en dresse alors le catalogue, en commençant par ceux qui «ont notre foi universelle, c'est-à-dire les déchaux ${ }^{48}{ }$.

\section{Voyages en Occident}

Pendant le second $\mathrm{xv}^{\mathrm{e}}$ siècle, deux groupes de voyageurs tchèques se dirigèrent également en Europe occidentale. Les deux sont étroitement liés à la politique étrangère de Georges de Poděbrady, roi de Bohême. Grâce à des traductions modernes, les deux voyages sont désormais relativement connus des milieux francophones ${ }^{49}$. Le premier, le texte laissé par l'écuyer Jaroslav, est le témoignage d'une mission purement diplomatique auprès de

45. Ibid., p. 81 .

46. Voire religions: Kabátník est aussi très tolérant envers les juifs de Terre sainte, peutêtre sous l'influence de son guide provenant de la communauté juive locale.

47. J. KoLÁR, «České znění Cesty do Jeruzaléma Martina Křivoústého... », p. 82.

48. Jan HasištejnskÝ zLobkovic, Putování..., p. 82.

49. "Le Journal de l'ambassade tchèque en France en 1464 par l'écuyer Jaroslav», éd. E. AdDE et M.NejEdLÝ, Annuaire-Bulletin de la SHS, année 2009, Paris, 2012, p. 53-117, où se trouvent des références à d'autres études sur ce sujet. Les deux textes sont aussi traduits
Louis XI, roi de France, en 1464; le second, celui de Venceslas (Václav) Šašek de Bîrkov, est un témoignage d'un membre de l'ambassade de Léon (Lev) de Rožmitál, beau-frère du roi de Bohême, effectuée en 1465-1467 pour des raisons plus complexes ${ }^{50}$

L'entreprise diplomatique de Léon de Rožmitál a en effet plusieurs dimensions et se distingue, entre autres, par un aspect non-négligeable de pèlerinage. Léon est présenté par Šašek comme un pieux catholique qui se rend à un pèlerinage chevaleresque. L'auteur participe aussi par son action à la construction de cette image ${ }^{51}$. L'ambassade tchèque prit la forme d'une série de visites des cours des souverains européens, organisées dans une ambiance d'hospitalité et selon les usages les plus distingués. Le but du voyage était en premier lieu de manifester la noblesse et le haut niveau du parent proche du roi calixtin qu'était Léon. Par ce biais, les représentants du royaume de Bohême tendaient à combattre la mauvaise image de la société tchèque, suspectée d'hérésie. Pour cette raison, Venceslas Šašek rappelle à plusieurs reprises que le voyage avait pour but le tombeau de saint Jacques à Compostelle ${ }^{52}$. Enfin, cette dimension est soulignée par la présence d'une liste d'indulgences pour la Terre sainte conservée avec le récit de Šašek.

dans le recueil de D.PÉrICARD-MÉa éd., De la Bohême jusqu'à Compostelle. Aux sources de l'idée d'union européenne, Biarritz, 2008, p. 75-114 (Jaroslav) et p. 139-342 (Václav Šašek). 50. Commentarius brevis et iucundus itineris, éd. K. Hrdina, Prague, 1951. Le récit ains que le voyage de Léon de Rožmitál ont attiré récemment l'attention des chercheurs à l'échelle européenne.Parmi les études les plus récentes, voir F.MichaUd-FréJAVILLE, «Dangereux Occident. Le voyage de Léon de Rožmitál jusqu’à Saint-Jacques de Compostelle (14651466)», Cahiers de Recherches Médiévales, 3 (1997), p. 57-69; W. PaRAvicinI, «Bericht und Dokument, Leo von Rožmitál unterwegs zu den Höfen Europas (1465-1466) «, Archiv für Kulturgeschichte, 92 (2010), p. 253-307. Le récit du voyage de 1465-1467 n', Arć für Kulurgeschichte, 92 (2010), p. 253-307. Le récit du voyage de 1465-1467 n' a pas été conservé dans sa forme originelle en langue tchèque du $x^{\circ}$ siècle. On ne connaît que sa traduction latine, faite en 1577 par Stanislav Pavlovský, futur évêque d'Olomouc, qui étai alors au service des descendants de Rožmitál. La participation du traducteur à la forme définitive du récit reste un thème de débat. $C f$. à ce propos F. Michaud-FréJAVILLE, «Le voyage du seigneur Léon de Rozmital en Occident, un apprentisage?», Voyages et voyageurs au Moyen Âge, Paris, 1996, p. 31-51; M. MalaNíkovÁ, «Ke vzniku cestopisu Václava Saǎ̌ka z Bířkova a možnostem jeho interpretace», Studia Historica Brunensia, 55 (2008), p. 21-33. Il reste malgré tout certain que l'ouvrage était interprété comme un voyage d'instruction dans le contexte du milieu du Xvi ${ }^{\mathrm{e}}$ siècle.

51. Un autre récit de ce voyage, écrit par Gabriel Tetzel, bourgeois de Nuremberg, qui participa lui-même au «tour» de Rožmitál, contraste avec la conception hérö̈que de l'ambassade qui ressort du récit du chevalier tchèque. Voir J.Schmeller éd., Des böhmischen Herrn Leo's von Rožmital Ritter-, Hof- und Pilger-Reise durch die Abendlande 1465-1467, Stuttgart, 1844, p. 143-196.

52. «Perspicimus ex litteris domini nostri te virum esse inclytum summoque genere natum, quamobrem si cupis, Compostellam usque te comitari volumus proque eo praemium nullum deposcimus» (Commentarius brevis..., p. 42) ; «Inde retro ad Divum Iacobum itinere converso Turones rediimus» (ibid., p. 51). 
Bien que l'ambassade ne se soit jamais rendue outre mer, il n'est pas à exclure que l'intention initiale était aussi de visiter la Palestine ${ }^{53}$

Alors que Venceslas Šašek est catholique (de même que le chef de l'ambassade), le récit de voyage de l'écuyer Jaroslav exprime la perspective utraquiste. Sa posture de partisan du calice est évidente malgré le caractère inachevé de son ouvrage. Le premier éditeur du récit, František Palacký, prit cette perspective pour «un rigorisme moral et religieux». Plus tard, cette interprétation a été révisée au profit d'une certaine incohérence des opinions de Jaroslav. D'un côté, ce dernier critique la tiédeur de la foi et, de l'autre, il ne formule pas d'objection contre une certaine liberté des mœurs dans les bains publics. Dans son récit, il est évident que la perception stéréotypée des nations n'empêche pas les relations de respect à l'intérieur du groupe social de la noblesse, ni les relations diplomatiques. Tandis qu'au contact du «menu peuple» Jaroslav vivait l'expérience d'être accusé d'hérésie, dans le milieu des grandes villes et de la haute aristocratie, ces rapports étaient beaucoup plus ambivalents ${ }^{54}$. C'est surtout la description de la ville de Constance que l'on peut considérer comme la manifestation la plus évidente de l'identité utraquiste de Jaroslav ${ }^{55}$ :

Le lendemain, le vendredi de la Saint-Barthélemy, nous fîmes quatre très longs miles jusqu'à Constance sur le Rhin, où les infâmes Allemands avaient brûlé le saint maître Jean Hus. Cette nuit-là, quand nous logeâmes là, le tonnerre gronda très fort. Toute la nuit les méchants prêtres firent sonner les cloches contre le tonnerre, surtout dans l'église Saint-Pierre, c'est-à-dire là où ils avaient condamné le saint maître Jean Hus à mort, etc.

Les épithètes, telles qu' «infâmes Allemands » ou «saint maître Jean Hus », sont suffisamment éloquentes. Le tonnerre est, lui, perçu comme une punition des bourreaux de Hus. En revanche l'emploi de cloches en guise de protection contre la foudre est considéré par Jaroslav, en bon utraquiste, comme une pratique superstitieuse. Ce n'est qu'au $\mathrm{XvI}^{\mathrm{e}}$ siècle que les protestants de Constance commencèrent à créer une culture mémorielle de Jean Hus; dès les années 1460, Jaroslav en avait déjà fait un élément de la mémoire collective des utraquistes ${ }^{56}$.

Malgré la méfiance de la théologie utraquiste envers les reliques, le récit de Jaroslav fait référence à ce phénomène à plusieurs reprises. Ce

53. J. SCHMELler éd., Des böhmischen Herrn Leo's von Rožmital..., p. 136-142.

54. B. MAŁYsZ, «,Mravní a nábožný rigorismus “? Vzájemné hodnocení Čechủ a cizinců v době poděbradské ve světle cestopisu panoše Jaroslava», Sborník prací pedagogické fakulty Masarykovy univerzity, r̆ada společenských věd, 23 (2009), p. 15-32.

55. «Le Journal de l'ambassade tchèque... », p. 115.

56. B. ZILYNSKÁ, «Reformační Kostnice a husovská tradice», dans R.NovotnÝ et P. Šámal éd., Zrození mýtu. Dva životy husitské epochy, Prague-Litomyšl, 2011, p. 329-341. n'est pourtant que la comparaison avec le récit de Šašek qui permet de voir la valeur différente attribuée aux reliques dans la représentation narrative des deux textes. La visite de Nuremberg en fournit un exemple idéal car les deux ambassades s'y arrêtèrent pour voir sa collection de reliques. L'écuyer Jaroslav aussi bien que Venceslas Šašek donnent une longue liste de ces restes sacrés. Jaroslav exprime une certaine méfiance envers leur authenticité et leur pouvoir («si ce qu'ils disent est vrai»), voire son indifférence («nous vîmes beaucoup d'autres choses curieuses, que je n'ai pas décrites ici, car cela ne me semble pas utile, etc. $\left.{ }^{57} »\right)$. En revanche, l'auteur catholique ne manque pas de vénérer ces reliques («Nous avons vénéré de nombreuses reliques de saints qu'on nous a montrées»). L'énumération qui suit est amplifiée de surcroît par des récits de miracles:

Puis nous avons vu: les chaînes des saints Pierre et Paul qui ont souffert pour le nom de Dieu, la lance qui a transpercé le divin côté du Christ. Nous l'avons observée avec attention, car les prêtres nous ont fait poser nos anneaux sur elle, nous disant que les gens atteints de coliques ou de douleurs trouvent là un remède immédiat et assuré ${ }^{58}$

L'historiographie a préféré reconstituer les circonstances politicodiplomatiques des deux ambassades, ce qui lui paraissait plus important que la vénération des reliques et la dénonciation des pratiques superstitieuses. Ces manifestations représentent pourtant des éléments clés de la construction identitaire et des représentations de l'auteur, et restent importantes également du point de vue politique. C'est particulièrement évident chez Šašek, qui avait l'intention de montrer Léon de Rožmitál, beau-frère du roi excommunié Georges de Poděbrady, comme un catholique fidèle. Son personnage est construit à travers son récit sur les motifs du pèlerin et chevalier dévot. À cette fin, l'auteur du récit n'hésite pas à recourir à des superlatifs que l'on peut également retrouver dans ses descriptions de reliques ${ }^{59}$. L'écuyer Jaroslav traduit plutôt le débat interconfessionnel; son récit contient néanmoins le caractère ambigu des stéréotypes confessionnels et nationaux.

57. «Le Journal de l'ambassade tchèque... », p. 93.

58. Commentarius brevis..., p. 13. Le pouvoir miraculeux des saintes reliques est aussi attesté par Šašek à propos d'autres lieux de pèlerinage, par exemple sur le tombeau de saint Thomas Becket à Cantorbéry (ibid., p. 34-35).

59. Par exemple: «Reliquiarum sacrarum, ut antea dixi, nullo in loco tantum simul numerum vidi. Quas mihi conscribere et annotare conanti dicebatur nequaquam possibile esse, ut eas omnes assignare possim, tantam enim earum vim esse, ut a duobus scribis per duas septimanas conscribi non queant.» (Ibid...., p. 37.) 
On peut donc diviser le corpus des récits de voyages médiévaux en langue tchèque en trois catégories. Tout d'abord, celle des traductions de Jean de Mandeville et de Marco Polo : le thème du voyage exotique les relie à d'autres ouvrages d'histoire et de fiction du tournant des XIV et $\mathrm{XV}^{\mathrm{e}}$ siècles.

La seconde catégorie, qui appartient aux années 1450-1500, est celle des récits de pèlerinage en Terre sainte, où se laissent observer des différences de perspective entre les auteurs en fonction de leur position religieuse. Bien que l'institution même du pèlerinage en Terre sainte transcendât les clivages confessionnels et que les auteurs tchèques respectassent les règles formelles du genre, le récit de voyage fournit parfois un medium pour leurs opinions. La confrontation des textes de Martin Křivoústý et de Jan Hasištejnský de Lobkovice et de leurs interprétations divergentes des pratiques liturgiques est un exemple, quoiqu'indirect, de polémique religieuse par l'intermédiaire de ce type de texte. L'utraquiste Křivoústý voyait dans la variété des rites de communion la confirmation de l'usage tchèque réformé; Hasištejnský, lui, soulignait avec force que, de tous ceux qui s'intitulaient chrétiens dans la ville de Jérusalem, seuls les catholiques étaient de véritables chrétiens. Dans les deux cas, le problème confessionnel propre à la Bohême apparaît en filigrane.Les mêmes actualisations socio-religieuses peuvent être discernées dans les incunables témoignant de la popularité du genre «viatique» et de la réception du fameux récit de Breydenbach dans le milieu tchèque à la fin du $\mathrm{XV}^{\mathrm{e}}$ et au début du XVI $\mathrm{I}^{\mathrm{e}}$ siècle.

$\mathrm{Au}$ troisième domaine appartiennent les deux récits de voyage en Occident. Écrits dans le contexte de la politique extérieure du « roi hérétique», Georges de Poděbrady, ils laissent voir deux postures différentes. L'appartenance confessionnelle de Venceslas Šašek de Bîrkov n'est pas explicite, et se dessine plutôt sur l'arrière-plan de sa construction de chevalier idéal, Léon de Rožmitál. L'écuyer Jaroslav, pour sa part, n'hésite pas à critiquer les autres (sans créer pour autant une image bipolaire), ce qui l'aide, en retour, à construire sa propre identité utraquiste.

Le problème de la confessionnalité dans les récits de voyage de Bohême représente sans aucun doute une piste non négligeable pour leur interprétation. L'identité, tant catholique qu'utraquiste, s'y introduisait selon une mesure différente à chaque fois bien que l'objectif essentiel fût d'instruire et de distraire le public. En ce sens, ces textes permettent de voir jusqu'à quel degré leurs auteurs adhèrent aux représentations de leur propre camp, et à quel point aussi les clivages religieux traversant la société tchèque s'avèrent plus flous qu'on ne les a parfois jugés ${ }^{60}$.

60. Cet article a été mené à bien grâce au soutien de l'Agence tchèque de la recherche GA CR) dans le cadre du projet P405/12/G148 «Les codes culturels et leurs transformations à l'époque hussite».
Vojtěch BAŽANT - Centrum medievistických studií AV ČR (Centre d'études médiévales), Prague.

Jaroslav SVÁTEK - Centrum medievistických studií AV ČR (Centre d'études médiévales), Prague.

Les récits de voyage médiévaux originaires de Bohême: produits d'une société confessionnalisée?

Cette étude analyse la question de l'identité confessionnelle dans la littérature de voyage au $\mathrm{XV}^{\text {e }}$ siècle en Bohême. Les clivages entre les différentes religions influencèrent ces textes et ceux-ci devinrent un medium pour commenter la question confessionnelle. Malgre la similarite des perception religieuses du monde entre les catholiques, les utraquistes et les membres de l'Unité des Frères (ainsi que de ceux dont l'identité confessionnelle n'est pas claire), les textes analysés reflètent des polémiques visant les autres groupes confessionnels.

$\mathrm{XV}$ e siècle - Bohême - confessionnalisation - pèlerinage - récit de voyage

Medieval Travelogues from Bohemia: Products of a Society Characterized by its Different Religious Communities?

This paper analyses the question of confessional identity in the Fifteenth century Bohemian travel literature. The religious division influenced these texts and they became a medium for their authors to comment on this question. Despite the similar religious perception of the world among the Catholics, the Utraquists or the members of the Unity of Brethren (and those of unclear religious identity), the texts we analyse reflect the polemics aimed at the other religious communities.

Bohemia - Fifteenth century - pilgrimage - religious communities travelogues 
